\title{
Gray Matter Increase Induced by Practice Correlates with Task-Specific Activation: A Combined Functional and Morphometric Magnetic Resonance Imaging Study
}

\author{
Rüdiger Ilg, ${ }^{1}$ Afra M. Wohlschläger, ${ }^{1,2,3}$ Christian Gaser, ${ }^{4}$ Yasmin Liebau, ${ }^{1}$ Ruth Dauner, ${ }^{5}$ Andreas Wöller, ${ }^{1}$ \\ Claus Zimmer, ${ }^{2}$ Josef Zihl, ${ }^{5,6}$ and Mark Mühlau ${ }^{1}$ \\ Departments of ${ }^{1}$ Neurology, ${ }^{2}$ Neuroradiology, and ${ }^{3}$ Nuclear Medicine, Technische Universität München, 81675 Munich, Germany, ${ }^{4}$ Department of \\ Psychiatry, University of Jena, 07743 Jena, Germany, ${ }^{5}$ Department of Psychology, Neuropsychology, Ludwig-Maximilians-Universität, 80802 Munich, \\ Germany, and ${ }^{6}$ Max-Planck-Institute of Psychiatry, Neuropsychology Research Group, 80804 Munich, Germany
}

The neurophysiological basis of practice-induced gray matter increase is unclear. To study the relationship of practice-induced gray matter changes and neural activation, we conducted a combined longitudinal functional and morphometric (voxel-based morphometry) magnetic resonance imaging (MRI) study on mirror reading. Compared with normal reading, mirror reading resulted in an activation of the dorsolateral occipital cortex, medial occipital cortex, superior parietal cortex, medial and dorsolateral prefrontal cortex, as well as anterior insula and cerebellum. Daily practice of 15 min for 2 weeks resulted in an increased performance of mirror reading. After correction for pure performance effects, we found a practice-related decrease of activation at the right superior parietal cortex and increase of activation at the right dorsal occipital cortex. The longitudinal voxel-based morphometry analysis yielded an increase of gray matter in the right dorsolateral occipital cortex that corresponded to the peak of mirror-reading-specific activation. This confirms that short-term gray matter signal increase corresponds to task-specific processing. We speculate that practice-related gray matter signal changes in MRI are primarily related to synaptic remodeling within specific processing areas.

Key words: mirror reading; practice; neural plasticity; gray matter changes; voxel-based morphometry; VBM; functional magnetic resonance imaging; fMRI

\section{Introduction}

In recent years, voxel-based morphometry (VBM) has proved a powerful method for the in vivo study of human brain structure in healthy and diseased subjects (Mechelli et al., 2005), yet the physiological basis of structural changes in VBM is incompletely understood. Although it is widely assumed that, in neurodegeneration, gray matter loss in VBM corresponds to neural loss (Baron et al., 2001; Thieben et al., 2002), the physiological basis of differences in regional gray matter (GM) in healthy subjects is unclear. However, several studies have shown that regional GM values are directly related to functional abilities (Maguire et al., 2000; Gaser and Schlaug, 2003; Mechelli et al., 2004). Moreover, an increase in GM can be induced by training and activation, which has been demonstrated for training of juggling for 3 months (Draganski et al., 2004), intensive studying for 3 months (Draganski et al., 2006), and repetitive transcranial magnetic stimulation (rTMS) for only $5 \mathrm{~d}$ (May et al., 2007). The relationship of induced GM changes and cerebral activation has not been investigated. Thereby, it is particularly unclear how induced GM

Received Dec. 27, 2007; revised Feb. 6, 2008; accepted Feb. 29, 2008.

This work was supported by the Gerhard u. Irmgard Schulz Fond (payment of subjects).

Correspondence should be addressed to Dr. Rüdiger Ilg, Department of Neurology, Klinikum Rechts der Isar,

Technische Universität München, Ismaninger Strasse 22, 81675 Munich, Germany. E-mail: ilg@|rz.tum.de.

D0I:10.1523/JNEUROSCI.5722-07.2008

Copyright $\odot 2008$ Society for Neuroscience $\quad$ 0270-6474/08/284210-06\$15.00/0 increase relates to task-specific processing. Aiming at this question, we performed a combined longitudinal structural (VBM) and functional magnetic resonance imaging (fMRI) study to examine the correlation of induced GM increase with (1) taskspecific activation (i.e., task-specific processing) and (2) practiceinduced changes of activation (i.e., practice effects).

Looking for a task that is sufficiently demanding to require substantial training, easy to quantify, and good to apply in the scanner, we decided to study the training of mirror reading, a complex procedural learning task that has been intensively studied. Longitudinal fMRI studies showed a shift of brain activation after successful training of mirror reading from parietal and occipital areas to temporal regions (Poldrack et al., 1998; Kassubek et al., 2001) or, at least, a decrease of activation in parietal areas and eye-movement-associated frontal areas (Kassubek et al., 2001).

\section{Materials and Methods}

Subjects. Thirty-eight (practice group, 20; control group, 18) volunteer male, healthy, right-handed (Edinburgh handedness inventory of practice group: mean, 88 ; SD, 11.55) subjects (mean age, 24 years; range, $20-32$ years) participated in the study. All subjects gave written informed consent before the experiment in accordance with the declaration of Helsinki. Two subjects of the practice group did not finish the study. One did not complete the 2 weeks of practice; the other produced motion artifacts in the second session. To ensure comparability of behavioral and 
imaging data, the behavioral data refer to the remaining 18 participants exclusively. Each of these remaining subjects was matched by a control (mean age, 25 years; range, 20-32 years).

Stimuli and experimental design. All 18 subjects of our final experimental group (mean age, 23 years; range, 20-27 years) practiced for 2 weeks (14 d) reading a mirrored " $15 \mathrm{~min}$ " magazine article per day. The articles were taken from popular German magazines covering various topics from sports to politics. Before as well as after the first and second week of practice, we measured the performance in reading that was defined by the time to read aloud standardized word lists. The word lists consisted of 50 normal (NW) or mirrored (MW) words that were balanced with respect to frequency of occurrence in German language and length (mean word length, 8.5 letters). Words were not repeated, i.e., each word list comprised different words. MRI was performed $1 \mathrm{~d}$ before and $1 \mathrm{~d}$ after the 2 weeks of practice, each day comprising two functional runs [echoplanar images (EPI)] and two morphometric scans [magnetization-prepared rapid acquisition gradient echo (MPRAGE)]. In the functional part of the experiment (blocked, self-paced design), subjects were instructed to silently read words (mirrored and normal words) that were presented on a projection screen in front of the scanner. The words were presented on slides containing three words (a so-called "triad," black letters on green background, one word below the other). MW and NW were matched for letter frequency. Each fMRI session comprised two runs at 12 blocks of the four basic conditions (NW and MW as well as two other conditions that will be analyzed in another context). The stimuli consisted of triads with NW (maximum, 156 triads) and MW (maximum, 156 triads). We randomized the conditions within runs, triads within conditions, and assignment of triads to conditions and runs. Subjects were instructed to silently read the presented words and press a button with their right index finger to trigger the presentation of the next triad as soon as they had deciphered all three words. On average, subjects read $177 \mathrm{MW}$ triads in the first session (i.e., before practice) and $281 \mathrm{MW}$ triads in the second session (i.e., after practice), as well as $551 \mathrm{NW}$ triads in the first session and $615 \mathrm{NW}$ triads in the second session. The 18 matched controls only underwent the $2 \times 2$ morphometric scans (on the same days as the respective practice subjects). Neither fMRI measurements nor practice of mirror reading was performed in the control group.

Behavioral data analysis. The performance of reading and mirror reading was measured by standardized word lists outside of the scanner (in words per minute) before practice (i.e., immediately before the first scan), after 1 week of practice (i.e., practice control), and after 2 weeks of practice (i.e., immediately before the second scan). Only words that were correctly deciphered were counted. Differences in performance and error rates before and after practice were analyzed by paired $t$ test for dependent samples.

Imaging parameters. fMRI data were acquired with a $1.5 \mathrm{~T}$ Siemens (Erlangen, Germany) Magnetom Symphony whole-body scanner using an eight-channel head coil. Multislice T2*-weighted EPIs were obtained from a gradient-echo sequence (plane, oblique axial; number of slices, 33; slice thickness, $4 \mathrm{~mm}$; interslice gap, $0.4 \mathrm{~mm}$; flip angle, $90^{\circ}$; in-plane resolution, $3.125 \times 3.125 \mathrm{~mm}^{2}$; field of view, $200 \mathrm{~mm}$; echo time, $50 \mathrm{~ms}$; repetition time, $3000 \mathrm{~ms}$ ). For VBM, we acquired two three-dimensional T1 MPRAGE sequences per scan (plane, sagittal; number of slices, 160; slice thickness, $1 \mathrm{~mm}$; voxel size, $1 \times 1 \times 1 \mathrm{~mm}^{3}$; flip angle, $15^{\circ}$; field of view, $256 \times 256 \mathrm{~mm}$; repetition time, $8.9 \mathrm{~ms}$; echo time, $3.93 \mathrm{~ms}$; inversion time, $800 \mathrm{~ms}$ ).

Statistical thresholds. As implemented in SPM (Statistical Parametric Mapping software; Wellcome Department of Imaging Neuroscience, London, UK; http://www.fil.ion.ucl.ac.uk), all corrections for multiple statistical tests are predicated on the theory of Gaussian random fields (Friston et al., 1996; Nichols and Hayasaka, 2003). We will refer to thresholds of voxel-level inference as height thresholds and to thresholds of cluster-level inference as extent thresholds. Applying an extension of SPM, VBM (http://dbm.neuro.uni-jena.de/), all extent thresholds had been corrected for anisotropy of smoothness, which is necessary especially for the analysis of VBM data (Ashburner and Friston, 2000; Hayasaka et al., 2004). For all analyses, we applied a height threshold of 0.001 uncorrected and an extent threshold of 0.05 corrected.

fMRI analysis. The fMRI analysis was performed with SPM5. The first

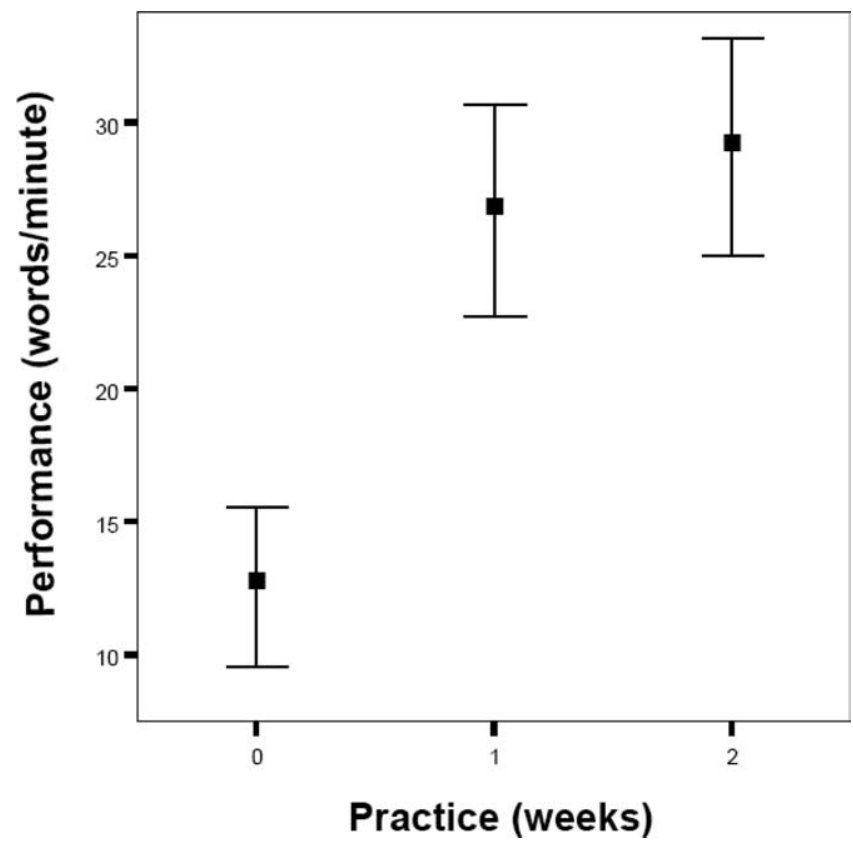

Figure 1. Practice-related performance increase. Practice resulted in a significant increase in mirror-reading performance (mean $\pm S D, p<0.001$ ).

three images of each run were discarded to allow equilibration of longitudinal magnetization. The preprocessing steps were (1) realigning and unwarping the images to correct for movement artifacts and related susceptibility artifacts, (2) coregistration of the anatomical to the functional images, (3) segmentation and normalizing of the anatomical image to the standard stereotactic space [Montreal Neurological Institute (MNI)], (4) application of normalization transformation to the functional images, and (5) smoothing with a Gaussian kernel of $8 \mathrm{~mm}$ for group analysis. Low-frequency components of data were removed by high-pass filtering. For each subject, images were subjected to a fixed effects analysis. At the group level, a random effects analysis was performed. The number of responses (i.e., the number of triads deciphered per block) was modeled as covariate to control for the number of attended word triads and performance-related effects. We regarded this a reasonable approximation of mirror-reading performance because the number of silently read mirror words in the scanner correlated significantly with the number of correctly deciphered mirror words outside of the scanner (Pearson's correlation coefficient, $0.86 ; p<0.001$ ).

In a first analysis, we focused on the overall activation of the task and therefore modeled all conditions versus baseline (data not reported). In the second analysis, we focused on activation specific for mirror reading (i.e., mirror reading vs normal reading). In the third analysis, we focused on the effects of practice on task-specific processing and compared activation related to mirror reading before and after practice.

The anatomical representation of the clusters was related to cytoarchitectonic probability maps as implemented in the SPM Anatomy Toolbox (Eickhoff et al., 2005).

$V B M$ analysis. Data processing of the morphological data was performed with VBM2 software, an extension of SPM2. Default settings were used. For the analysis of longitudinal data, VBM2 coregisters the images of one subject to the first image. The normalization parameters are calculated only for the first image and then applied to the remaining images to prevent that regional differences between the images are removed simply because of scan-specific spatial normalization. Moreover, VBM2 applies the "optimized" protocol (Good et al., 2001) and additionally removes inhomogeneities in signal intensity between subsequent scans. Furthermore, a hidden Markov random field model (Cuadra et al., 2005) is applied to minimize the noise level by "removing" isolated voxels of one tissue class, which are unlikely to be member of this tissue class. The resulting GM images were smoothed with a Gaussian kernel of 8 


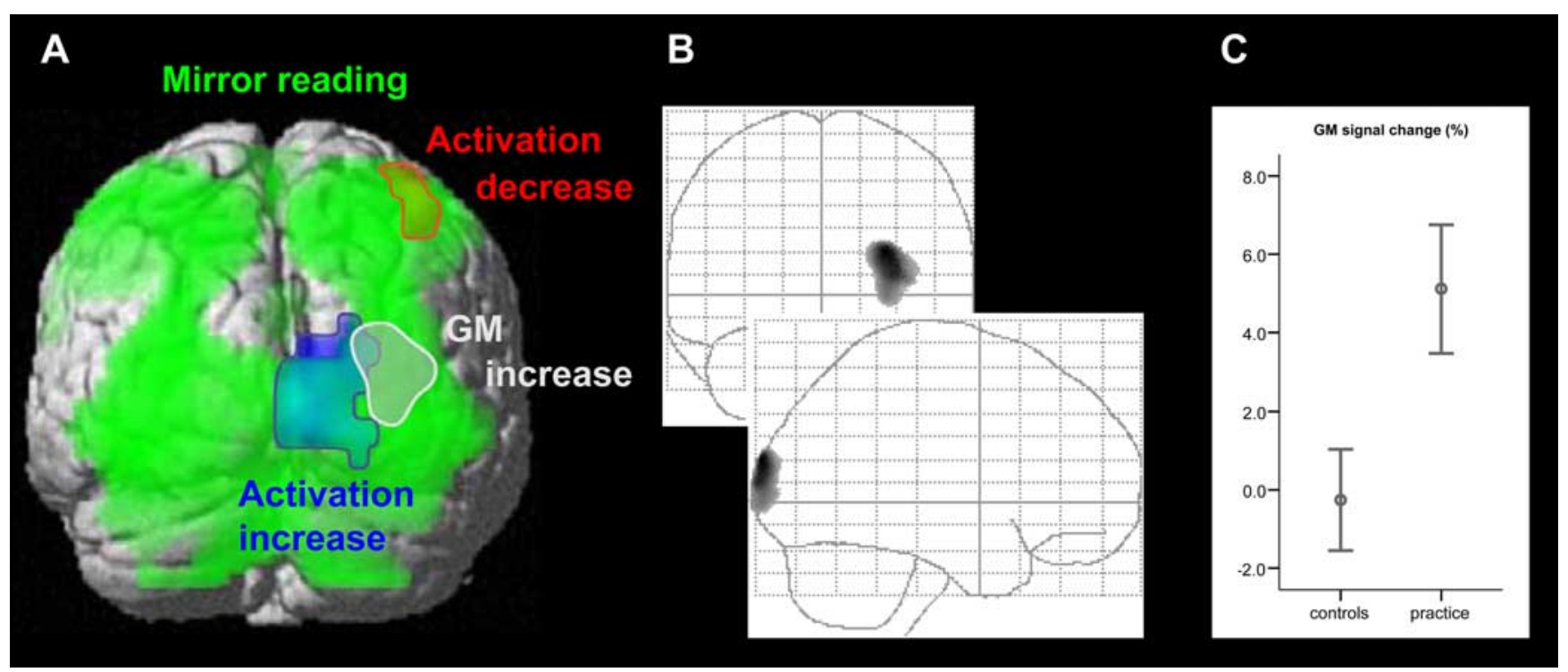

Figure 2. Practice-related changes in activation and gray matter. $A$, Clusters of activation and GM changes are displayed at a height threshold of 0.001 uncorrected and an extent threshold of 0.05 corrected. Mirror-reading-specific activation (green), practice-related decrease of activation (red), practice-related increase of activation (blue), and practice-related increase of GM (gray) are superimposed on a surface-rendered MNI template. Compared with normal reading, mirror reading resulted in a differential activation of bilateral dorsal occipital lobe (inferior, middle, and superior occipital gyrus), occipitotemporal cortex (fusiform gyrus), superior parietal cortex (bilateral superior parietal lobule, left intraparietal sulcus, bilateral precuneus, and left somatosensory cortex), medial and dorsolateral prefrontal cortex (left presupplementary motor area, right middle cingulate cortex, left frontal eye field, and bilateral precentral gyrus), right anterior insula, and cerebellum. The comparison of GM before and after practice shows a significant increase in GM in a subset of the regions (gray) activated in the right occipital cortex during mirror reading (green). $\boldsymbol{B}$, The middle plot shows the maximum intensity projection of the GM increase. C, The right plot displays the GM signal change (as a percentage) of all voxels of the cluster (we divided the individual mean of the $G M$ values of all voxels of the resulting cluster after practice by the individual mean of the $G M$ values of the same voxels before practice; error bars indicate mean and SEM). The mean relative $G M$ signal increase within the reported cluster was $5.1 \%$ (SEM, $1.6 \%$ ) in the practice group and $-0.3 \%$ in the control group (SEM, $1.3 \%$ ).

$\mathrm{mm}$. To analyze only voxels with sufficient GM and to avoid possible edge effects around the borders between GM and WM as well as between GM and CSF, we included only voxels with a GM value $>0.1$ (range, $0.0-1.0$ ). In addition, we performed the same analysis with the control group and, to certainly exclude that GM changes identified by our VBM analysis resulted from shifts of MRI parameters over time, an interaction analysis of group and time. Here, we applied a small-volume correction for the GM changes identified in the practice group. Finally, visual "sliceby-slice" comparison of the mean structural image (all anatomical scans of the practice group) and the mean functional image (all functional scans of the practice group) showed an exact correspondence so that a coregistration of the images derived from the different modalities was not necessary.

\section{Results}

\section{Behavioral data}

Analysis of reading performance before and after practice revealed a significant increase in mirror-reading performance from $12.6 \pm 6.0$ to $26.6 \pm 8.0$ words $/ \mathrm{min}$ after the first week of practice and to $29.0 \pm 8.2$ words/min after the second week of practice (mean performance increase, $160 \pm 80 \%$; range, +46 to $+333 \%$; $p<0.001)$. Performance increase during the first week of practice was significantly higher than during the second week of practice (Fig. 1). The error rate decreased significantly from $7.9 \pm 5.6$ to $1.8 \pm 2.3 \%$ after the first week of practice $(p<0.001)$ and further to $0.9 \pm 1.4 \%$ after the second week of practice (not significant, $p=0.149)$. Normal reading performance did not change significantly $(92.7 \pm 24.0$ vs $97.3 \pm 23.3$ words $/ \mathrm{min} ; p=0.158)$.

\section{fMRI data}

The first analysis (overall activation) revealed a widespread activation pattern predominantly involving the cerebellum, primary visual cortex as well as motor- and language-related areas (data not shown).

The second analysis aimed at identifying activation specifi- cally associated with mirror reading. Mirror reading compared with normal reading resulted in a broad activation of the dorsal occipital lobe bilaterally (inferior, middle, and superior occipital gyrus), occipitotemporal cortex (bilateral fusiform gyrus), parietal cortex (bilateral superior parietal lobule, left intraparietal sulcus, bilateral precuneus, and left somatosensory cortex), medial and dorsolateral prefrontal cortex (left presupplementary motor area, right middle cingulate cortex, left frontal eye field, and bilateral precentral gyrus), as well as right anterior insula and cerebellum (Fig. $2 \mathrm{~A}$, in green; Table 1).

The third analysis aimed at identifying effects of practice on task-specific processing. The comparison of mirror-reading related activation (fMRI) before and after practice revealed an increase of activation in the right occipital cortex and left thalamus (Fig. 2A, blue; Table 2) and a decrease of activation in the right superior parietal cortex (Fig. $2 \mathrm{~A}$, red; Table 2).

\section{VBM data}

The comparison of GM before and after practice showed no decrease but a significant increase in a subset of the regions activated in the right occipital cortex (Fig. $2 A, B$, in gray; Table 2 ). The center of the cluster corresponded well to the peak of mirror-reading-specific activation (distance, $18 \mathrm{~mm}$ ). The analysis of the control group revealed no significant changes in GM over time even when reducing the height threshold to 0.01 uncorrected. For the cluster of GM increase within the practice group, the interaction analysis (group $\times$ time; smallvolume corrected) also revealed a significant increase of GM (peak voxel, $p=0.00003$, uncorrected; $p=0.007$, corrected; cluster, $p=0.01$, corrected), indicating that the signal changes over time could not be attributed to shifts of MRI parameters over time. 
Table 1. Mirror reading versus normal reading

\begin{tabular}{|c|c|c|c|c|c|c|}
\hline \multirow[b]{2}{*}{ Region } & \multirow[b]{2}{*}{ Localization of peak voxels } & \multirow[b]{2}{*}{ Hemisphere } & \multicolumn{3}{|c|}{ MNI coordinates (mm) } & \multirow[b]{2}{*}{$Z$ values (peak voxel) } \\
\hline & & & $x$ & $y$ & $Z$ & \\
\hline \multirow[t]{9}{*}{ Dorsal occipital } & \multirow[t]{4}{*}{ Inferior occipital gyrus (BA 18/19/37) } & \multirow[t]{2}{*}{$\mathrm{R}$} & 36 & -84 & -3 & 5.97 \\
\hline & & & 42 & -75 & -12 & 5.52 \\
\hline & & \multirow[t]{2}{*}{ L } & -42 & -60 & -12 & 5.86 \\
\hline & & & -36 & -87 & -12 & 5.46 \\
\hline & \multirow[t]{3}{*}{ Middle occipital gyrus (BA 18/19) } & $\mathrm{R}$ & 36 & -84 & 9 & 5.69 \\
\hline & & $\mathrm{L}$ & -30 & -84 & 12 & 5.76 \\
\hline & & & -30 & -93 & 18 & 5.23 \\
\hline & \multirow[t]{2}{*}{ Superior occipital gyrus (BA 19/7) } & $\mathrm{R}$ & 27 & -69 & 27 & 5.60 \\
\hline & & $\mathrm{L}$ & -24 & -69 & 33 & 5.86 \\
\hline \multirow[t]{4}{*}{ Occipitotemporal } & \multirow[t]{4}{*}{ Fusiform gyrus (BA 19/37) } & \multirow[t]{2}{*}{$\mathrm{L}$} & -36 & -48 & -21 & 5.75 \\
\hline & & & -36 & -69 & -12 & 5.56 \\
\hline & & \multirow[t]{2}{*}{$\mathrm{R}$} & 39 & -69 & -18 & 5.71 \\
\hline & & & 33 & -48 & -18 & 5.68 \\
\hline \multirow[t]{8}{*}{ Parietal } & \multirow[t]{4}{*}{ Superior/inferior parietal lobule (BA 7/40) } & \multirow[t]{2}{*}{$\mathrm{L}$} & -18 & -63 & 39 & 5.66 \\
\hline & & & -24 & -66 & 54 & 5.27 \\
\hline & & \multirow[t]{2}{*}{ R } & 27 & -57 & 45 & 5.45 \\
\hline & & & 24 & -66 & 51 & 5.28 \\
\hline & Intraparietal sulcus (BA 40) & $\mathrm{L}$ & -45 & -39 & 42 & 5.47 \\
\hline & \multirow[t]{2}{*}{ Precuneus (BA7) } & $\mathrm{L}$ & -15 & -75 & 45 & 5.18 \\
\hline & & $\mathrm{R}$ & 12 & -69 & 45 & 5.24 \\
\hline & Somatosensory cortex (BA 2) & $\mathrm{L}$ & -42 & -42 & 54 & 5.05 \\
\hline \multirow[t]{6}{*}{ Frontal } & \multirow[t]{2}{*}{ Pre-SMA (BA 6) } & \multirow[t]{2}{*}{$L$} & -6 & 12 & 48 & 5.55 \\
\hline & & & -3 & 3 & 54 & 5.45 \\
\hline & Middle cingulate cortex (BA 24/32) & $\mathrm{R}$ & 9 & 18 & 39 & 5.16 \\
\hline & Frontal eye field (BA 6) & $\mathrm{L}$ & -30 & -6 & 51 & 5.45 \\
\hline & \multirow[t]{2}{*}{ Precentral gyrus (BA 6/9) } & \multirow[t]{2}{*}{$L>R$} & -51 & -3 & 48 & 5.29 \\
\hline & & & 51 & 9 & 36 & 5.12 \\
\hline \multirow[t]{2}{*}{ Limbic } & \multirow[t]{2}{*}{ Insula lobe } & \multirow[t]{2}{*}{$\mathrm{R}$} & 36 & 24 & 9 & 5.21 \\
\hline & & & 36 & 21 & 0 & 5.12 \\
\hline \multirow[t]{2}{*}{ Cerebellum } & Posterior lobe & $R>L$ & 15 & -72 & -21 & 5.11 \\
\hline & Vermis (declive/pyramis) & $\mathrm{R}$ & 9 & -72 & -21 & 5.33 \\
\hline
\end{tabular}

Note that the table reports all peak voxels surviving a corrected height threshold of $p<0.05$. BA, Brodmann area.

\section{Table 2. Practice effects}

\begin{tabular}{|c|c|c|c|c|c|c|c|}
\hline \multirow[b]{2}{*}{ Region } & \multirow[b]{2}{*}{ Localization of peak voxels } & \multirow[b]{2}{*}{ Hemisphere } & \multicolumn{3}{|c|}{ MNI coordinates (mm) } & \multirow[b]{2}{*}{$Z$ value (peak voxel) } & \multirow[b]{2}{*}{ Corrected $p$ value (cluster) } \\
\hline & & & $x$ & $y$ & $z$ & & \\
\hline \multicolumn{8}{|c|}{ Decrease of activation (fMRI) } \\
\hline Parietal & Superior parietal (BA 1/2, hiP2) & $\mathrm{R}$ & 42 & -39 & 54 & 4.35 & 0.017 \\
\hline \multicolumn{8}{|c|}{ Increase of activation (fMRI) } \\
\hline Occipital & Dorsal occipital (BA 17/18) & $\mathrm{R}$ & 9 & -90 & 0 & 4.24 & $<0.001$ \\
\hline Thalamus & Anterior & $\mathrm{L}$ & -9 & -6 & 12 & 4.01 & 0.039 \\
\hline \multicolumn{8}{|c|}{ Gray matter signal increase (VBM) } \\
\hline Occipital & Dorsal occipital (BA 18/19) & $\mathrm{R}$ & 29 & -97 & 19 & $5.17^{*}$ & 0.003 \\
\hline
\end{tabular}

BA, Brodmann area; hiP2, human intraparietal area 2. ${ }^{*} p$ corrected, 0.029 .

\section{Discussion}

\section{Behavioral data: improvement in} mirror-reading performance

The practice resulted in a highly significant increase of mirrorreading performance (Fig. 1). Because performance of normal reading did not change significantly, improvement in mirror reading can be attributed to specific practice and not to a general adaptation to the task. Although we acquired only three measurements of reading performance, the obtained data indicate that the effect of practice was initially pronounced and declined with time. This time course corresponds to behavioral data that showed that visual skill learning in a mirror-reading task follows a negatively accelerated power function (Kolers, 1975, 1976; Poldrack and Gabrieli, 2001).

\section{Task-specific activation of mirror reading}

Compared with normal reading, mirror reading primarily resulted in a robust parietal and occipitotemporal activation
(Fig. 2A). This pattern is in line with several imaging studies that reported activation of both the dorsal visual and, to a lesser extent, ventral visual stream in spatial transformations of written language (Goebel et al., 1998; Poldrack et al., 1998; Dong et al., 2000; Poldrack and Gabrieli, 2001; MochizukiKawai et al., 2006). Poldrack et al. (1998) were the first to examine the practice effects in mirror reading with fMRI. Comparing reading of mirrored and normal text, they found widespread bilateral activation of occipital, parietal, and inferior temporal regions, which primarily corresponds to our data (Poldrack et al., 1998). Dong et al. used Japanese kana words that are read top to bottom (whether mirrored or not) to minimize oculomotor effects and, by this, focused on the visuospatial transformation and cognitive processing of mirror words. Again, widespread activation of predominantly occipital and parietal areas was found, which corresponds well to our results (Dong et al., 2000). 


\section{Practice-related changes of activation}

Functional MRI revealed a practice-related decrease of activation in the right superior parietal cortex and an increase of activation at the right dorsal occipital cortex. Several studies applying different training protocols analyzed practice influences on activation during mirror reading. The results have been, at least in part, inconsistent. Analyzing differences between three sessions of mirror reading within 2 weeks, Poldrack et al. (1998) found practice-related decrease in the right superior parietal cortex that corresponds to the practice-related decrease of activation in our experiment. Furthermore, they reported a practice-related decrease in the posterior occipital cortex bilaterally, right pulvinar, right inferior cerebellum, and right inferior temporal cortex. The reported deactivation included parts of the inferior and middle occipital gyri bilaterally, which showed an increased activation in our study. Moreover, they found areas of increased activation at the precuneus, left superior parietal cortex, left fusiform gyrus, right superior temporal cortex, and right cerebellum. However, this study differed from ours in essential aspects, such as training, paradigm, and statistical modeling of performance. Following a different approach, Kassubek et al. (2001) obtained two fMRI scans on 2 consecutive days, one before and one after practice with mirror reading for several hours before the second fMRI scan on the next day. In accordance with our study, they found a decrease of activation within right superior parietal areas. Kassubek et al. proposed that the observed decrease of activation in the superior parietal cortex was the result of an "increase in the efficiency of specialized mental transformation processes" that lead to a "reduction in the effort and time required to decode mirror-reversed letters and hold them in visuospatial working memory" ( $p 216$ ). Following this argumentation, the increase of activation in higher visual areas of the dorsal occipital lobe in our study could reflect an increase of mirror-reading-specific processing, whereas the right superior parietal activation before training could reflect the initially higher effort of visuospatial transformation. This shift of activation from parietal to higherorder visual processing areas is in line with the assumption of a shift from visuospatial transformation to direct recognition of mirror words (Poldrack et al., 1998).

\section{Practice-related gray matter increase}

This is the first study that related GM increase induced by practice to respective task-specific activation. We could demonstrate that the GM increase in the right dorsolateral occipital cortex corresponded to the site of the peak activation during mirror reading and increase of activation by practice. The mean relative GM signal change of 5\% in the practice group was in the range of the results reported by Draganski et al. (2004). GM increase was only found within the right hemisphere, which corresponds to the activation pattern and findings of other functional imaging studies. Both the activation during mirror reading and practicerelated decrease (Kassubek et al., 2001), as well as the activation during reading of kana mirror words (Dong et al., 2000) were pronounced in the right superior parietal cortex, indicating a right hemispheric dominance for visuospatial transformation. A right hemispheric dominance of visuospatial transformation is further supported by tasks on mental rotation of objects and characters (Harris et al., 2000; Podzebenko et al., 2002).

With respect to the nature of inducible GM increase, the following mechanisms have been regarded as possible (May and Gaser, 2006): simple change in cell size of neurons or glial cells, genesis of glial cells (or even neurons), as well as changes in the intracortical axonal architecture (i.e., most likely synaptogen- esis), which is thought to represent the morphological basis of long-term learning (Bailey et al., 2004). From a functional perspective, GM increase induced by practice that has been reported so far (Draganski et al., 2004, 2006) could correspond (1) to mere previous activation (i.e., most likely mechanisms such as changes in cell size) or (2) to intracortical axonal remodeling. The fact that, in humans, GM increase can be induced by rTMS over a period as short as $5 \mathrm{~d}$ has been interpreted as support for fast adjusting neuronal systems such as spine and synapse turnover, whereas slow evolving mechanisms such as neuronal or glial cell genesis seem to be less likely (May et al., 2007). The GM increase in our study was located at the site of maximal task-specific activation and not at the site of maximal overall activation, which challenges the notion that GM increase is a result of mere previous activation (e.g., change in cell size, genesis of cells). In contrast, our results point toward an intracortical axonal remodeling for several reasons. First, because we could induce GM increase by a practice of merely $15 \mathrm{~min} / \mathrm{d}$, it seems more likely that GM increase corresponds to changes in task-specific processing than to a surplus of sustained activation. Second, the site of GM increase did not correspond to the site of overall activation. Third, the GM increase identified by our study was located in an area that is most likely associated with processing of mirror words.

The association of task-specific activation and corresponding GM increase supports hypotheses on an important role of structural neuroplasticity in the processing of novel information (Chambers et al., 2004). We speculate that short-term practice-induced changes in GM are related to intracortical remodeling.

\section{References}

Ashburner J, Friston KJ (2000) Voxel-based morphometry-the methods. NeuroImage 11:805-821.

Bailey CH, Kandel ER, Si K (2004) The persistence of long-term memory: a molecular approach to self-sustaining changes in learning-induced synaptic growth. Neuron 44:49-57.

Baron JC, Chetelat G, Desgranges B, Perchey G, Landeau B, de la Sayette V, Eustache F (2001) In vivo mapping of gray matter loss with voxel-based morphometry in mild Alzheimer's disease. NeuroImage 14:298-309.

Chambers RA, Potenza MN, Hoffman RE, Miranker W (2004) Simulated apoptosis/neurogenesis regulates learning and memory capabilities of adaptive neural networks. Neuropsychopharmacology 29:747-758.

Cuadra MB, Cammoun L, Butz T, Cuisenaire O, Thiran JP (2005) Comparison and validation of tissue modelization and statistical classification methods in T1-weighted MR brain images. IEEE Trans Med Imaging 24:1548-1565.

Dong Y, Fukuyama H, Honda M, Okada T, Hanakawa T, Nakamura K, Nagahama Y, Nagamine T, Konishi J, Shibasaki H (2000) Essential role of the right superior parietal cortex in Japanese kana mirror reading: an fMRI study. Brain 123:790-799.

Draganski B, Gaser C, Busch V, Schuierer G, Bogdahn U, May A (2004) Neuroplasticity: changes in grey matter induced by training. Nature 427:311-312.

Draganski B, Gaser C, Kempermann G, Kuhn HG, Winkler J, Buchel C, May A (2006) Temporal and spatial dynamics of brain structure changes during extensive learning. J Neurosci 26:6314-6317.

Eickhoff SB, Stephan KE, Mohlberg H, Grefkes C, Fink GR, Amunts K, Zilles K (2005) A new SPM toolbox for combining probabilistic cytoarchitectonic maps and functional imaging data. NeuroImage 25:1325-1335.

Friston KJ, Holmes A, Poline JB, Price CJ, Frith CD (1996) Detecting activations in PET and fMRI: levels of inference and power. NeuroImage 4:223-235.

Gaser C, Schlaug G (2003) Brain structures differ between musicians and non-musicians. J Neurosci 23:9240-9245.

Goebel R, Linden DE, Lanfermann H, Zanella FE, Singer W (1998) Functional imaging of mirror and inverse reading reveals separate coactivated networks for oculomotion and spatial transformations. NeuroReport 9:713-719. 
Good CD, Johnsrude IS, Ashburner J, Henson RN, Friston KJ, Frackowiak RS (2001) A voxel-based morphometric study of ageing in 465 normal adult human brains. NeuroImage 14:21-36.

Harris IM, Egan GF, Sonkkila C, Tochon-Danguy HJ, Paxinos G, Watson JD (2000) Selective right parietal lobe activation during mental rotation: a parametric PET study. Brain 123:65-73.

Hayasaka S, Phan KL, Liberzon I, Worsley KJ, Nichols TE (2004) Nonstationary cluster-size inference with random field and permutation methods. NeuroImage 22:676-687.

Kassubek J, Schmidtke K, Kimmig H, Lucking CH, Greenlee MW (2001) Changes in cortical activation during mirror reading before and after training: an fMRI study of procedural learning. Brain Res Cogn Brain Res 10:207-217.

Kolers P (1975) Memorial consequences of automized encoding. J Exp Psychol Hum Learn Mem 104:689-701.

Kolers P (1976) Reading a year later. J Exp Psychol Hum Learn Mem 2:554-565.

Maguire EA, Gadian DG, Johnsrude IS, Good CD, Ashburner J, Frackowiak RS, Frith CD (2000) Navigation-related structural change in the hippocampi of taxi drivers. Proc Natl Acad Sci USA 97:4398-4403.

May A, Gaser C (2006) Magnetic resonance-based morphometry: a window into structural plasticity of the brain. Curr Opin Neurol 19:407-411.

May A, Hajak G, Ganssbauer S, Steffens T, Langguth B, Kleinjung T, Eichhammer P (2007) Structural brain alterations following 5 days of intervention: dynamic aspects of neuroplasticity. Cereb Cortex 17:205-210.
Mechelli A, Crinion JT, Noppeney U, O’Doherty J, Ashburner J, Frackowiak RS, Price CJ (2004) Neurolinguistics: structural plasticity in the bilingual brain. Nature 431:757.

Mechelli A, Price CJ, Friston KJ, Ashburner J (2005) Voxel-based morphometry of the human brain: methods and applications. Curr Med Imaging Rev 1:1-9.

Mochizuki-Kawai H, Tsukiura T, Mochizuki S, Kawamura M (2006) Learning-related changes of brain activation in the visual ventral stream: an fMRI study of mirror reading skill. Brain Res 1122:154-160.

Nichols T, Hayasaka S (2003) Controlling the familywise error rate in functional neuroimaging: a comparative review. Stat Methods Med Res 12:419-446.

Podzebenko K, Egan GF, Watson JD (2002) Widespread dorsal stream activation during a parametric mental rotation task, revealed with functional magnetic resonance imaging. NeuroImage 15:547-558.

Poldrack RA, Gabrieli JD (2001) Characterizing the neural mechanisms of skill learning and repetition priming: evidence from mirror reading. Brain 124:67-82.

Poldrack RA, Desmond JE, Glover GH, Gabrieli JD (1998) The neural basis of visual skill learning: an fMRI study of mirror reading. Cereb Cortex $8: 1-10$.

Thieben MJ, Duggins AJ, Good CD, Gomes L, Mahant N, Richards F, McCusker E, Frackowiak RS (2002) The distribution of structural neuropathology in pre-clinical Huntington's disease. Brain 125:1815-1828. 